\title{
Hacia un nuevo esquema de empleo público. Un sueño visionario...
}

\author{
Jon Azúa Mendía *
}

\section{Introducción}

El presente artículo obedece al intento de reflexionar, una vez más, sobre el complejo mundo del empleo público, su percepción por la Sociedad en que está inmerso y a cuyo servicio justifica su existencia en el marco de un tiempo de innovación permanente generador de incertidumbres, dificultades y -sobre todo- oportunidades de futuro

Para acercarnos a su análisis, recurriremos a la combinación de la experiencia vivida desde una rica, joven y compleja Administración (Administración Pública Vasca) y a las perspectivas de cambio que el entorno ofrece. Así, empezaremos por situar el EMPLEO PÚBLICO en el centro de todo proceso de reforma de la Administración, reinvención de gobierno o simplemente gestión normalizada de la Cosa Pública. A continuación analizaremos el proceso de cambio entre el hoy y el mañana en al menos cuatro escenarios condicionantes del empleo público: a) la propia Administración Pública, b) la Europa y el mundo abierto en el que habremos de insertarnos, c) el mundo del ciudadano-consumidor-cliente y su demanda ante las Administraciones Públicas y d) el propio mundo de los recursos humanos.

Finalmente, tras contrastar la evolución prevista en los mencionados escenarios con los mecanismos de adaptación, resistencia e innovación del propio empleo público (tanto el empleador como el empleado y el ciudadano) sugerimos una visión deseada y esperanzada hacia la que nos gustaría se dirigieran los esfuerzos de todos al servicio de una nueva Sociedad.

\section{Empleo Público y Reforma de la Administración}

Hoy no existe político, administrador, líder académico, comunicador o simple ciudadano que se precie que no abandere una inevitable reforma de las Administraciones Públicas. Son múltiples las razones que la sugieren, mayor el número de iniciativas propuestas y escasos los procesos estratégicos que, liderados por la cabeza, se llevan a la práctica con plena convicción tanto del compromiso asumido como de la firme voluntad de culminarlos.

Y es aquí donde, entre diseños de visiones y procesos estratégicos, libros blancos y verdes, compromisos parlamentarios e instrumentos ejecutivos, terminamos en el origen de toda reforma: los Recursos Humanos. Y es aquí, por tanto, donde suele empezar y terminar la voluntad y/o compromiso de REINVENTAR EL GOBIERNO.

El empleo público se convierte o bien en la excusa para abandonar un laborioso y complejo trabajo ("ni los funcionarios ni los sindicatos colaborarán...", "Los partidos políticos no aceptarán la profesionalización de la Admón...", "todo lo que hagamos será intrascendente mientras no suprimamos el carácter permanente de la relación contractual...", etc.) o en el centro exclusivo de atención ("hemos congelado plantillas...", "hemos reducido el número de asesores y cargos públicos...", "hemos contenido la masa salarial...", etc.).

Todos ellos son elementos clave de la realidad reformadora pero no son ni los únicos ni constituyen la base real del cambio inevitable

A partir de aquí, el debate está servido. ¿Qué características y atributos habrán de corresponder a un EMPLEO PÚBLICO acorde con un nuevo mundo por alcanzar? ¿Acaso un modelo ancestral de función pública que respondía a un concepto de EstadoNación-Sociedad-Ciudadanos-Servicio determinados en un contexto socio-económico específico puede convivir con una nueva concepción de la Administración en un mundo de individuos cambiantes? ¿Cómo y a qué ritmo puede y debe adaptarse el actual empleo público al aún incierto horizonte de futuro que en todo caso conllevará modelos de trabajo incapaces de garantizar el pleno empleo? Son éstas y otras muchas las preguntas básicas que tanto la Sociedad como los dirigentes políticos y administradores públicos -sin olvidar la inevitable reflexión de los propios empleados públicos- debemos hacernos y no sólo para comprobar su complejidad y renunciar al futuro, sino para aproximarnos con firmeza y decisión a un proceso comprometido con la búsqueda de soluciones. 
Para todo ello, resulta imprescindible acercarnos al análisis de los condicionantes del entorno pero no sin antes desprendernos -en la medida de lo posible- de anclajes al pasado y al presente; de tópicos y simplismos que nos llevarían al inmovilismo, antes de iniciar el ejercicio reflexivo en clave positiva.

\section{Condicionantes del Entorno y Expectativas de Futuro}

Concentremos nuestra atención en el Empleo Público (los gobernantes elegidos y/o designados; los funcionarios; los contratados laborales en cualquiera de sus acepciones; sus promotores y representantes; los marcos legales existentes; las condiciones del ejercicio del trabajo en el seno de la Administración y sus relaciones con Terceros) y tratemos de interpretarlo, de manera simultánea, con todos y cada uno de los distintos condicionantes del entorno, en su panorama actual y los escenarios previstos de futuro, de modo que seamos capaces de concebir las líneas maestras de la visión que generaremos en la conclusión de este artículo.

\subsection{Escenarios interrelacionados}

Utilicemos el esquema que aparece en la figura $n^{0} 1$. En él observamos que el Empleo Público se configura como motor, causa y consecuencia, a la vez, de otros cuatro elementos duales -hoy y mañana- que condicionan el entorno de manera interrelacionada:

* La propia Administración Pública

* La Sociedad globalizada y doméstica en el marco de una nueva Europa por construir

* El ciudadano, consumidor y cliente

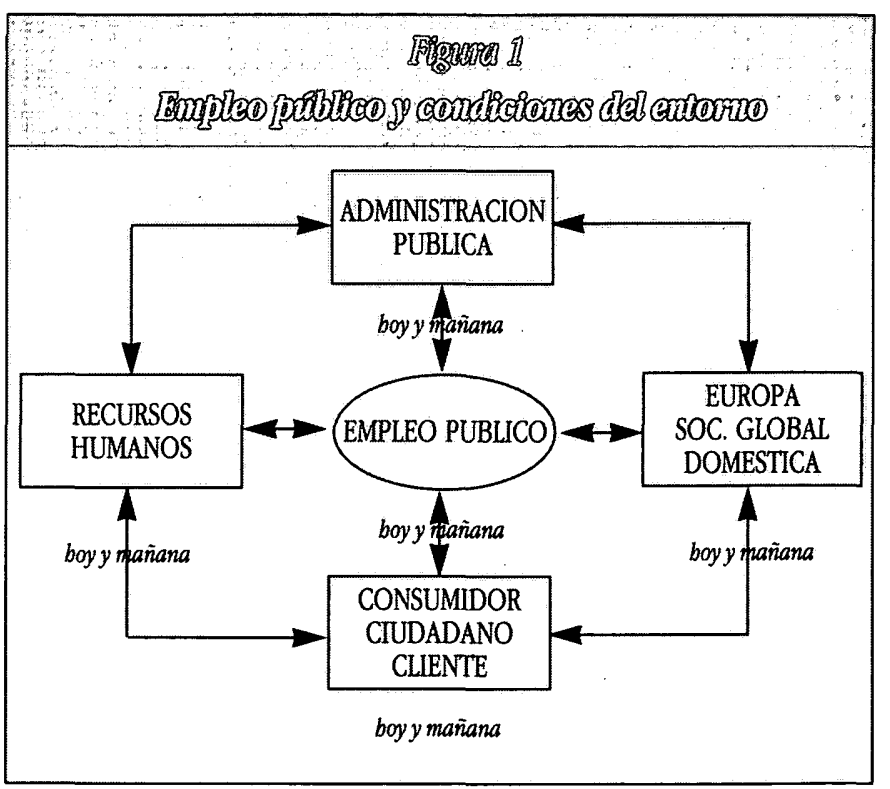

* Los recursos humanos en sí mismos

* El empleo público.

No siendo, sin embargo, objeto del presente artículo profundizar en cada uno de los elementos y sus escenarios de futuro, nos limitaremos a avanzar algunas ideas que permitan aproximarnos al enfoque deseado y su reflejo en el empleo desde las Administraciones Públicas al servicio de la Sociedad.

\subsection{Características actuales de cada uno de los Escenarios}

De una manera sintética podríamos reflejar los escenarios actuales en los "fotogramas" siguientes:

A. ADMINISTRACIÓN PÚBLICA. Sometida a una doble presión (una, positiva que le hace objeto de una demanda creciente de nuevos servicios y espacios de atención al ciudadano, y otra, negativa que le desacredita y pide su redimensionamiento a la baja así como pautas de eficacia y transparencia que pretenden denunciar como inexistentes o precarias) que le sitúa en el centro de todos los males que aquejan a la Sociedad como responsable directo de su solución, a la vez que le exige su retirada propiciadora de un supuesto liderazgo y protagonismo de la llamada Sociedad Civil; aboga por una reducción de la apelación fiscal y tributaria para la financiación de los servicios a la vez que no parece aceptar tasas o extracostes asociados con pagos de uso, unidos a una feroz crítica a su gestión, cuando no a la cualificación y dedicación de sus responsables y/o protagonistas.

B. EUROPA Y LA SOCIEDAD GLOBAL. Una Europa en construcción que ha agotado el éxito del objetivo '93 (El Mercado Interior) y se ha estancado en un Maastricht inalcanzable, poco comprendido y de dudosa ilusión. Su concepción política, soportada en criterios económicos cuantificables y en un organigrama político artificial carente de coherencia con procesos lógicos de toma de decisiones con participación ciudadana y respeto a la soberanía popular que inspiran los principios de la tradicional democracia europea, alejada del ciudadano y de todo control e impulso popular, se muestra incapaz de alumbrar ni liderazgo ni soluciones. Por contra, no parece evidenciarse alternativa alguna. Y esa Europa, dentro de una llamada Sociedad Globalizada que tampoco satisface la difícil demanda de un desarrollo socioeconómico armónico garantizador de un necesario Estado social de bienestar capaz de resolver la amenaza tecnología-desempleo, no hace sino agravar el deseado diseño de un nuevo modelo de inspiración e ilusión POLÍTICA, acompañado de ritmos económicos.

C. CIIENTE - CONSUMIDOR - CIUDADANO. Un hombremujer ciudadano(a) exigente, instruido, preocupado más por sus demandas personales -en una sociedad cada vez más individualista y encerrada en su propia "burbuja de seguridad"- que por los principios, ideales y compromisos, poco temerosa de involuciones democráticas que en su mayoría no han conocido ni padecido. Un hombre-mujer que separa las fronteras entre su triple papel conjunto ante el poder público como cliente, consumidor, ciudadano y, en algunos casos, acentuado por su propia condición de miembro de la cada vez más amplia franja de empleado público en su más amplia concepción. 


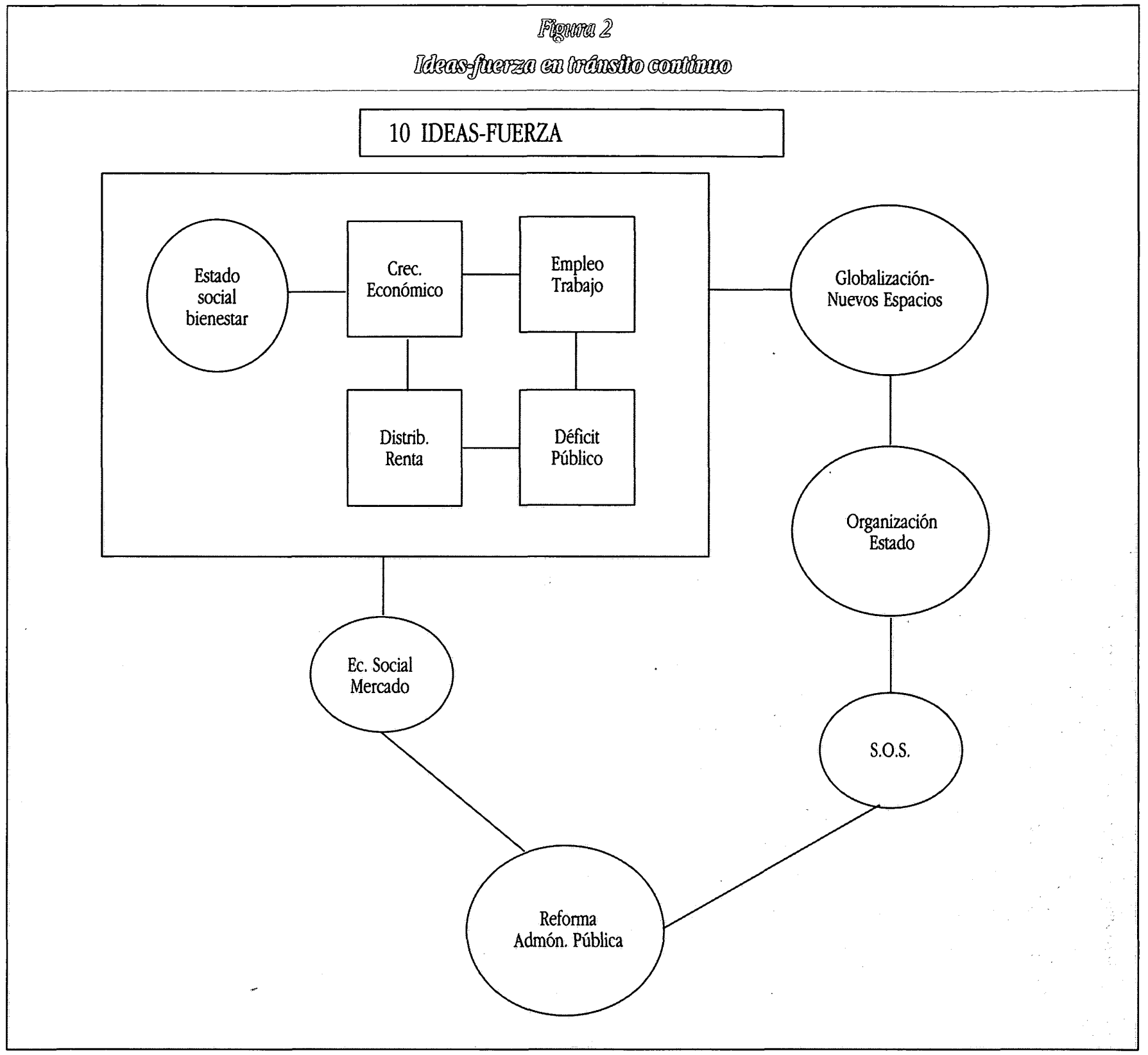

D. RECURSOS HUMANOS. El mundo de los recursos humanos que nos afecta no puede limitarse al empleado público sino que habrá de incluir, de manera especial, a las capas dirigentes (altos cargos y gobernantes), a los parlamentarios, concejales y conjunto de personas relacionadas tanto con estos colectivos como con el resto de los poderes públicos sin olvidar a los dirigentes de los partidos políticos y de los Sindicatos. Cada individuo, cada organización vive sus propias inquietudes, sus propios objetivos, sus propios mecanismos de selección, promoción y separación de personas tanto internos como en el conjunto de las Administraciones Públicas, en la medida en que dispongan de poder real para acceder a la toma de decisiones, bien de manera directa o indirecta, y -en todo caso- mediante el control de los procesos señalados. Individuos, por tanto, con su propia percepción de la Sociedad y de su evolución, de la Administra- ción, de su papel y trabajo, etc. Individuos que gozan de su propia satisfacción o insatisfacción en su trabajo, poseen su propia escala de valores, sus propios compromisos, su propio sentido de la solidaridad y su acción ante terceros. Ven, viven y protagonizan el cambio o, en su defecto, ejercitan la resistencia al mismo. Y es en este escenario en el que habremos de movernos destacando un colectivo: dirigentes, líderes, jefes y responsables. (De momento basta con señalarlos sin precisar términos...). Su actitud, dedicación, formación, compromisos, alternativas personales, capacidad de motivación y liderazgo, así como su adaptación a nuevos escenarios posibles y/o previstos será la clave del buen o mal funcionamiento del Empleo Público y su reforma.

E. EMPLEO PÚBLICO. El empleado público, en sus diferentes condiciones, se ve cada vez más protegido (de hecho) ante una sociedad dual -los parados y los que tienen empleo-, a la 
vez que más desprestigiado desde un punto de vista de opinión, al menos publicada. Profesional cada vez más y mejor formado para el desempeño de un servicio público que cada vez se parece menos a aquél para el que fue contratado, normalmente tras un intenso proceso de selección/oposición. Añade la inquietud, más verbal que real, de cambios o incluso pérdida de empleo ante las reformas por abordar. En todo caso, vive en organizaciones cambiantes, lentas, complejas y mixtas (unas áreas de gran interés, impacto público, responsabilidad y dedicación intensas; otras estériles, estáticas, poco eficaces y desconocidas incluso por sus propios jefes); representado, a su vez, por organizaciones sindicales obligadas a emprender su propia reforma, sumidas en sus propias ineficacias, procesos de ajuste, credibilidad social y, en su caso, bajo el impulso político de Partidos, también necesitados de su propia actualización.

\subsection{Su "filtro" a través de unas tendencias de futuro}

Diseñar un escenario futuro no resulta fácil. Quizás podamos compartir diagnósticos de situación pero aventurarnos con un acuerdo en lo futurible puede ser pedir demasiado. No obstante, la labor de un dirigente exige identificar visiones y fijar estrategias compartibles y compartidas- para alcanzar los objetivos que se propongan. Y para ello utilizaremos, aquí, tres referencias (podríamos recurrir a otras muchas pero tan sólo se pretende reflexionar sobre un posible punto de llegada y no acertar en el punto final único) cuyo uso conjunto y libre nos permitirá trasladar los escenarios descritos en los puntos anteriores hacia una opción del mañana.

Las referencias elegidas son:

a). Una crítica pesimista que parte de la tesis de la imposible solución de los problemas reales que aquejan a la Sociedad y a sus Administraciones Públicas, debido a que ni hay interés real en enfrentar los problemas, ni el largo sacrificio requerido en el empeño será aceptado ni por los responsables ni por sus protagonistas y, sobre todo, porque los plazos dados a los gobernantes para afrontarlos son demasiado cortos ${ }^{1}$.

b). Una especial preocupación por la actitud de los actores principales (gobernantes y empleados públicos) quienes, satisfechos por su posición privilegiada, desde una óptica y actitud corporativas, no están dispuestos a esforzarse en beneficio de otros y, sin saberlo, en el de ellos mismos en el largo plazo².

c). Una proyección de tendencias estratégicas desde una visión del marketing y la demanda social basada en la concepción individuo-cliente-ciudadano que permite apostar por líneas de comportamiento de la Sociedad en los próximos años ${ }^{3}$.

Con estas referencias, combinadas de una manera tal que permita su adaptación al objeto del presente trabajo, podemos resumir en 10 IDEAS-FUERZA los elementos/escenarios de cambio que condicionarán el nuevo EMPLEO PÚBLICO y que quedan reflejados en la figura $n^{\circ} 2$.

\section{Un nuevo Estado Social de Bienestar}

Uno de los mayores retos de los gobiernos de hoy y de mañana, y en especial de aquellos encuadrados en el llamado primer mundo, es garantizar la financiación y aplicación de un modelo de bienestar compatible con las posibilidades reales de nuestra economía y, sobre todo, con el compromiso de solidaridad necesario para superar la marginación. Marginación social, cultural, sanitaria, educativa y prioridades y límites obligados de atención y un adecuado esquema de contribución y financiación.

\section{Crecimiento económico, productividad y genera- ción de riqueza}

El hoy y el mañana no admiten una separación temporal e/o ideológica entre lo que debe ser primero si la creación de riqueza o su distribución. Estamos "condenados" a hacer casi todo a la vez. Todo crecimiento económico no vinculado a una productividad real y enfocada a la economía real generadora de un stock de riqueza y empleo a largo plazo será incapaz de garantizar un Estado que, a su vez, propicie un desarrollo económico armónico para toda la Comunidad. Sin este complejo mixto, no habrá futuro ilusionador.

\section{La justa y equitativa distribución de la renta}

Justicia y equidad vuelven a cobrar especial relevancia. No son ya objeto de debate ideológico sino elementos consustanciales al ejercicio del poder. Como elementos objetivos que son, obligan a la reformulación de políticas, sistemas y actitudes de gobiernos y Administraciones.

\section{Empleo, trabajo y paro}

La realidad es tozuda: el modelo socioeconómico actual es fórmula ensayada que resulta insuficiente. Una solución que sea coherente incapaz de superar el paro conocido y, sobre todo, el esperado. Toda con el resto de las diez ideas fuerza que aquí reseñamos pasa por un redefinición de dos conceptos que parecen confundirse y que tienen muy poco que ver en la Sociedad actual: Empleo y Trabajo. Las sociedades occidentales disponen de mucho trabajo excedente que puede reducir de forma considerable el paro. Sin embargo, son incapaces de ofrecer empleo para desarrollar la demanda de trabajo. Con precios y condiciones de empleo actuales -y más si pretende crearse desde el sector público-, el sistema económico -actual y globalizado- no puede acometer la creación de empleo deseada. Esa nueva definición, inmersa en un sustancial cambio en el modelo y otros conceptos parcialmente equivocados como la globalización de la economía y su carácter mítico, posibilitaría alternativas de solución.

\section{El déficit público}

Con o sin voluntad política y/o social de emprender una reforma de las Administraciones Públicas, el déficit público se erige como un indicador incuestionable que obligará al cambio. Un déficit excesivo y, sobre todo, creciente, impide cualquier política económica seria y rigurosa que ofrezca expectativas a los ciudadanos, a la vez que la vía fiscal como recurso de financiación mayoritaria se verá limitada con fuerza por la propia presión y sensibilidad sociales.

\section{Economia social de mercado}

Los cinco conceptos anteriores conllevan un marco preferente e incuestionable al futuro en el que se desarrollará la acción de 
los diferentes gobiernos: la economía social de mercado. Hoy han quedado superados los debates ideológicos de fondo entre alternativas socialismo-capitalismo. Este nuevo punto de encuentro, por contra, tiene aún asignaturas pendientes: el debate humanista, el rol del ciudadano, la aplicación real de la subsidiariedad y la solidaridad, el reconocimiento y aplicación de los derechos de las personas y de los pueblos, la organización del poder político y sus Administraciones garantes del modelo global, la reformulación de valores y la propia eficacia/eficiencia de su funcionamiento, incrementando la participación democrática y social tanto en la toma de decisiones como en el control de las mismas.

\section{La organización del Estado}

El Estado-nación ha llegado a su fin. Ha vivido tímidamente la generación de alternativas que le sobrevivan; no es capaz -ni quiere- propiciarlas sino más bien se empeña en obstaculizarlas y no sabe dar paso a una nueva fórmula alternativa. Se resiste a alumbrar el futuro y exagera las ventajas de su pasado. Pero, en todo caso, lo que está fuera de cuestión es la inevitabilidad de su transformación en un doble enfoque -dinámico y simultáneohacia la creación de bloques supraestatales y hacia la regionalización ("tribalización" que diría Peter DRUCKER) trasladando a nuevos espacios y ámbitos la toma de decisiones. Este proceso conlleva no sólo nuevos conceptos y actores sino una nueva sistemática de trabajo y oferta de servicios públicos.

\section{Globalización y nuevos espacios multidomésticos interdependientes}

Muy unido (iconsecuencia o causa?) al punto anterior aparece una nueva modalidad de aproximación a la realidad económica. La ola inicial de la globalización absoluta, de la mano de un imparable proceso de "federalización de la economía", da lugar a una compacta interrelación de sistemas autónomos interdependientes que parecen conjugar la dimensión adecuada para el tratamiento de los problemas, generan el apoyo y consenso suficiente entre valores culturales compartidos, aportan esquemas institucionales "completos", proporcionan participación y control democráticos suficientes y parecen movilizar recursos e intenciones en una misma dirección con visiones de las llamadas ÁREAS HOMOGÉNEAS que, como áreas base, posibilitan una nueva manera de superar conceptos de independencia desde proyectos interrelacionados compartibles desde el ejercicio pleno de autogobierno. Elemento clave en torno al cual se desarrollarán los movimientos y procesos de avance de la Sociedad, en lo político y en lo económico.

\section{La modernización y reforma de la Administración}

El conjunto de IDEAS-FUERZA hasta aquí recogido conlleva una modernización y reforma de las Administraciones Públicas, no como un punto final de llegada (cierto) sino como un proceso estratégico de mejora continua hacia un futuro, visionario, (incierto todavía hoy) que permita adaptar la res publica a las demandas cambiantes de la Sociedad. Este proceso no admite ni atajos ni simplicidades. Se trata de un camino largo y complejo que requiere una fuerte dosis de imaginación y coraje políticos. Sus soluciones no son únicas y sus ritmos habrán de marcarlos los líderes sociales y políticos y cada Sociedad en su momento.

\section{S.O.S. (Save our Society): Democracia y Ecologia}

Finalmente, el hombre (la mujer) y sus organizaciones son cada ecología. El eslogan "salvar la sociedad" cobra cada vez más fuerza se exprese como se exprese. Hoy no hay decisión de consecuencias económicas, sociales y, por supuesto, política que no lo contemple como parte inherente al propio proceso decisorio. Estas consideraciones generan un ciudadano, cliente, consumidor, cada día más consciente de la importancia de la democracia y cada vez más formado, exigente y vigilante lo que, a su vez, supone nuevas maneras de trabajar, nuevas organizaciones, nuevos contenidos de los empleos, nuevas fórmulas de financiación, de prestación de servicios y de participación y representación políticas. Y, cómo no, detrás de todo esto, está el EMPLEO PÚBLICO. Volvemos al principio y centro de la cuestión.

\section{4. ¿Y babrán de cambiar sus dirigentes...?}

Si hemos hecho un largo recorrido hasta aquí para acercarnos a los cambios que se están produciendo en la sociedad y que condicionarán el futuro del empleo público, coincidiremos en la inevitabilidad de propiciar nuevos dirigentes con nuevas actitudes y aptitudes, nuevas escuelas de dirección de trabajo y nuevos estilos de relación humana en el seno de las organizaciones públicas que conforman el conjunto de la Administración.

Dirigir no es tarea fácil ni está al alcance de todos. Se puede ser un extraordinario profesional, un fiel militante político o sindical, un riguroso y serio ciudadano, poseer un excelente curriculum-vitae -cosas todas ellas valiosas y positivas- pero necesariamente adecuadas para dirigir. El incierto y cambiante mundo que pesará sobre toda organización pública exige de dirigentes responsables capaces de conjugar, al menos, una serie de cualidades próximas al siguiente escenario:

"VISIÓN DE FUTURO que le permita imaginar lo inimaginable para propiciar la fijación de objetivos LARGOPLACISTAS superadores de legislaturas cortas, inspirado en una profunda ETICA personal que se traslade al conjunto de sus actuaciones y organización, con un amplio sentido de RESPONSABIIIDAD SOCIAL que le lleve a pensar, de manera sistemática y automática en TERCEROS, con capacidad probada para ASUMIR RIESGOS -personales y politicos-, desde una firme posición de EQUIIIBRIO EMOCIONAL, capaz de convivir con la tensión y presiones múltiples, generador y motivador de EQUIPOS comprometidos con PROYECTOS especificos de los que ellos mismos formen parte, desde una actitud de FORMACIÓN PERMANENTE, con capacidad para dejarse sorprender por lo novedoso e incierto, HETERODOXO en su voluntad de ruptura de inercias y saber conjugar la esquizofrenia de COOPERAR-COMPARTIR-COMPETIR con todos como elemento básico de avance".

Cualidades que exigen, a la vez, "estar siempre en movimiento" OYENDO AL CIUDADANO.

Como podemos observar, tarea difícil que exige el primer y mayor compromiso de la Sociedad y de sus partidos políticos y sindicatos: propiciar esquemas de selección, formación, promo- 
ción y separación de lideres y dirigentes cualificados para una nueva Administración Pública. A partir de aqui, la reforma del EMPLEO PÚBLICO no sería especialmente dificil.

\section{Un sueño visionario: Hacia un nuevo esquema de Empleo Público}

Si avanzar un cambio en el esquema de empleo público resulta complejo, hacerlo tras lo comentado hasta aquí en el presente artículo lo es aún más. No obstante, trataré de adelantar algunas "tendencias visionarias" que propicien la reflexión para su logro.

Comencemos por señalar que, como toda Reforma, la modernización y reforma del empleo público no es una acción estática ni única sino que se encuentra inmersa en un proceso de mejora continua que debe impulsarse con el mayor empeño, voluntad y dedicación posibles, provocando -desde el cambio actitudinal de la dirección- el resto de innovaciones necesarias atemperadas por las demandas reales de la Sociedad, en cada momento. Muchos son los procesos en marcha. Yo he tenido la suerte de participar a lo largo de casi quince años en algunos de ellos y algunas de las recomendaciones de mi aportación "visionaria" no son sino fruto de un intenso, enriquecedor y compartido proceso de debate y trabajo en el seno de la "Comisión para la racionalización y mejora de la Administración" impulsado por el Gobierno Vasco ${ }^{4}$.

Hechas estas observaciones, un esquema de futuro pasaría por los siguientes elementos:

1. Redefinir un esquema completo de empleo público en el marco de una gestión integrada de los recursos bumanos al servicio de la Administración.

2. Toda concepción del sistema contractual del servidor público requiere la asunción de la demanda de una nueva Sociedad, la adecuación previa de la organización de la Administración Pública a esa realidad y la implantación simultánea de sistemas alternativos de prestación de servicios públicos 5 .

3. Suprimir el carácter permanente e indefinido de la relación contractual del servidor público con la Administración, en el marco de una reforma integral que garantice el libre acceso al empleo, su transparencia, posibilite una formación y adaptación permanente a nuevos servicios, propicie movilidad y flexibilidad laborales, introduzca sistemas retributivos variables y diferenciados atendiendo a contenidos/esfuerzos/resultados reales de trabajo y establezca mecanismos objetivos de renovación contractual, acompañada de promoción individualizada y selectiva, incorporando en la evaluación mecanismos "sabáticos" favorecedores de planes específicos de formación.

4. Simplificación de estructuras organizativas y delimitación de niveles politicos y de gestión en la estructura directiva configurando un nivel de directivos públicos profesionales sujetos a regímenes especiales orientados a la realidad del mercado, bajo la regulación de status y códigos de conducta durante y después del ejercicio de su responsabilidad, desconcentrando la gestión de los recursos humanos asignados a las estructuras organizativas minimizadas.

5. Reformular los esquemas de formación en las Administraciones Públicas concentrando esfuerzos en necesidades reales para el trabajador/trabajo concreto a realizar, introduciendo programas de avance general en períodos sabáticos determinados.

6. Comprometer al personal al servicio de las Administraciones Públicas en el proceso de reforma previsto.

\section{Conclusión}

El cambio es inevitable. De lo que se trata es de dirigirlo hacia un escenario deseable. Aquí reside su complejidad y el verdadeo reto.

Propiciar un nuevo esquema de empleo público exige, antes de nada, "comprender y compartir el futuro", "ilusionarse con él e iniciar el duro y largo recorrido". Para ello, lo primero, se requiere voluntad política para liderar el cambio que empezará -y aquí reside la clave del éxito o fracaso de la tarea- en sus dirigentes. Si no lo intentamos nunca llegaremos a saber si la oposición corporativa era real o no. Corresponde, por tanto, a los partidos políicos empeñarse en la apuesta decidida por un sistema de futuro de selección, formación, promoción, control y separación de los responsables de la dirección y gestión de las Administraciones Públicas desde cuyo poder habrán de llevar a cabo su sueño y compromiso de transformar una Sociedad encaminada hacia un nuevo futuro deseado. A partir de ahí, el conjunto del sistema irá cambiando, de forma positiva, a medida que la propia Sociedad lo demande.

\section{Notas}

\footnotetext{
" Ingeniero Industrial y "master" por IESE. Ha sido Vice-Lehendakari y Consejero de Industria del Gobiemo Vasco durante la última legislatura. Con anterioridad había sido Consejero de Trabajo, Sanidad y Seguridad Social, y Secretario del Gobiemo. Fue Diputado de Promoción y Desarrollo Económico de la Diputación Foral de Vizcaya. En el ámbito privado, y entre otras actividades, ha sido Consejero-Director General de la Bolsa de Bilbao.

${ }^{1}$ Paul Krugman. The Age of Diminished Expectations.
}

${ }^{2}$ K. GalBraITH. The Culture of Contentment.

${ }^{3}$ Popcorn Talent Bank \& Trends. The Popcom Report.

4 "CORAME. Estrategia para la modemización y reforma de la Administración Pública Vasca. $1993^{n}$

${ }^{5}$ D. OSBORNE y Ted GAEBLER. 36 formas de prestar el servicio público. 